\title{
Cupriavius pauculus causing a respiratory tract infection in a post-kidney-transplant patient:A firstly described rare clinical case
}

\begin{abstract}
Cupriavidus pauculus is a gram-negative aerobic bacillus usually isolated from bottled mineral water, water from ultrafiltration systems in hospital setting and medical devices. Infections in humans are rare but usually severe, with most described cases of bacteremia, pneumonia, meningitis and sepsis. We describe a rare case of a Cupriavidus pauculus infection in a post-kidney-transplant context. A 60-year-old man, with Chronic Kidney Disease due to chronic glomerulonephritis, initiated hemodialysis in 1991 and received a cadaveric-donor kidney transplant in 1997. The graft functioned until 2008, when he returned to hemodialysis. After 2 years, he transited to Peritoneal Dialysis because of vascular access failure. He remained on PD for 7 years and received a new kidney transplant in 2018 after Encapsulating Peritoneal Sclerosis (EPS). The graft never gained function, so he remained peritoneal-dialysis-dependent. A few days after transplantation, peritonitis ensued (Enteroccocus faecium, Serratia marcescens). Even after Tenckhoff catheter removal, the clinical condition deteriorated, with need for continuous hemodiafiltration. The diagnose of a cecum perforation was made followed by hemicolectomy. Due to persisting respiratory and abdominal infections despite antibiotic regimens, and no kidney graft function, nephrectomy of the graft was performed, suspending immunosuppressive drugs. Later, a Cupriavidus pauculus was isolated in sputum, confirmed in two samples. Bacterial examination of the dialysis unit water system and equipment as well as of the ventilator used by the patient was negative. To our knowledge, this is the first case of a respiratory tract infection associated with Cupriavidus pauculus described in a post-kidneytransplant patient.
\end{abstract}

Volume 8 Issue I - 2020

\author{
David Fiel,' Rita Calça, ${ }^{2}$ Eunice Cacheira, ${ }^{2}$ \\ Nuno Rombo, ${ }^{3}$ Sara Querido, ${ }^{2}$ Célia \\ Nascimento, ${ }^{2}$ Cristina Jorge, ${ }^{2}$ André \\ Weigert, ${ }^{2}$ Rita Birne, ${ }^{2}$ Belarmino Clemente, ${ }^{3}$ \\ António Martinho, ${ }^{3}$ Cristina Toscano, ${ }^{4}$ \\ Margarida Bruges, ${ }^{2}$ Beatriz Malvar,' \\ Domingos Machado ${ }^{2}$ \\ 'Nephrology Department, Hospital do Espírito Santo, Évora, \\ Portugal \\ ${ }^{2}$ Nephrology Department, Hospital de Santa Cruz, CHLO, \\ Lisboa, Portugal \\ ${ }^{3}$ Surgery Department, Hospital de Santa Cruz, CHLO, Lisboa, \\ Portugal \\ ${ }^{4}$ Clinical Pathology Department, Hospital Egas Moniz, CHLO, \\ Lisboa, Portugal
}

Correspondence: David Carvalho Fiel, Hospital do Espírito Santo de Évora-Serviço de Nefrologia, Largo Senhor da Pobreza, 7000-8I I Évora, Portugal, Email davidcarvalhofie@gmail.com

Received: January 16, 2020 | Published: February 07, 2020

Keywords: kidney, transplant, respiratory infection, cupriavidus, pauculus

Abbreviations: $\mathrm{CKD}$, chronic kidney disease; $\mathrm{PD}$, peritoneal dialysis; ATG, antithymocyte globulin; MMF, micophenolate mofetil; PDN, prednisolone; ICU, intensive care unit; US, ultrasound; PDN, prednisolone; CVVHDF, continuous venovenous hemodiafiltration; DGF, delayed graft function; HD, hemodialysis; KT, kidney transplant

\section{Introduction}

Initially described by Yabuuchi in 1995 and previously known as CDC group IV C-2, Ralstonia, and later Wautersia, the genus Cupriavidus refers to ubiquitous, Gram-negative, aerobic, non-sporeforming mesophilic motile bacilli. They are catalase and oxidase positive, non-fermentative on MacConkey agar, oxidize glucose, and degrade nitrate. Colonies are round, smooth, convex, and nonpigmented. ${ }^{1}$ Widely distributed in nature, specially in water and soil, isolation has occurred in environmental samples of pool water, groundwater, tap and bottled mineral water when suspected to be a potential source of contamination in the clinical setting. ${ }^{2-8}$ The genus Cupriavidus contains 13 species, being C. pauculus historically implicated in the majority of the, nevertheless rare, infections. Both immunocompromised patients (with underlying comorbidities such as Chronic Kidney Disease (CKD), hematologic malignancies and Acquired Immunodeficiency Syndrome) and immunocompetent patients have been reported with infection, either in community or nosocomial setting., ${ }^{4,5}, 9$

These agents can act as opportunistic pathogens in a hospital setting and can cause outbreaks, especially in intensive care units. ${ }^{2}$ Although few reports have been published in the literature, there have been described cases of pneumonia, peritonitis, tenosynovitis, meningitis and bloodstream infections, specially in association with intravascular catheters and other medical devices. Other clinical described sources are hydrotherapy pools, nebulizers, ultrafiltrated water, thermoregulator reservoir water of extra-corporeal membrane oxygenation systems (ECMO) and invasive ventilators. ${ }^{3-5,10-12}$ The incidence of Cupriavidus spp. infections is unknown and may be underreported due to misidentification on many automated laboratory platforms. ${ }^{13}$ We present a rare case of a Cupriavidus pauculus infection in a postkidney-transplant context.

\section{Presentation of case}

A 60-year-old man with history of CKD due to presumed chronic glomerulonephritis (no kidney biopsy preformed) initiated hemodialysis (HD) in 1991 until 1997, when he received a cadavericdonor kidney transplant (KT) implanted in his right iliac fossa. The graft functioned until 2008, when he returned to HD. After 2 years, he 
transited to peritoneal dialysis (PD) because of vascular access failure and remained in the technique for 7 years, period when he developed Encapsulating Peritoneal Sclerosis (EPS) with progressively decaying of dialytic adequacy. Being referred as a priority patient, he received a second KT in May of 2018: 64-year-old donor after circulatory death, 4 HLA (Human leukocyte antigen) mismatches (1 in A, 2 in $\mathrm{B}, 1$ in DR), cold ischemia time of $22 \mathrm{~h} 10 \mathrm{~m}$, panel-reactive antibody (PRA) of $25 \%$, no preformed anti-HLA antibodies, induction immunosuppressive therapy with Antithymocyte globulin (ATG), Micophenolate Mofetil (MMF) and Prednisolone (PDN) followed by Tacrolimus. The surgical procedure was convoluted due to previous left iliofemoral thrombosis (first graft was still implanted in his right iliac fossa) and presence of petrous External Iliac Vein, therefore venous anastomosis was performed in his left Common Iliac Vein. Slow graft reperfusion was observed. Despite normal ultrasound (US), without any perfusion or obstructive anomalies, and renal scintigraphy with a delayed excretion of the tubular secretion agent, suggesting Acute Tubular Necrosis (ATN), the graft never gained function, so he remained PD dependent.

A few days after transplantation, peritonitis ensued (Enteroccocus faecium and Serratia marcescens were isolated in peritoneal effluent), for which he was medicated with directed antibiotic therapy. Even after Tenckoff catheter removal, the clinical condition deteriorated with severe abdominal pain and hemodynamic instability with need for continuous venovenous hemodiafiltration (CVVHDF) and the diagnose of a spontaneous cecum perforation, confirmed by abdominal tomography, was made and followed by hemicolectomy and colostomy construction. Our patient developed respiratory failure with need of invasive ventilation and was transferred to the Intensive Care Unite (ICU). A nosocomial respiratory tract infection was diagnosed and due to persisting abdominal infections despite directed antibiotic regimens, with no kidney graft function, nephrectomy of the graft was performed, with suspension of immunosuppressive drugs. Later, a Cupriavidus pauculus was isolated in sputum, confirmed in two samples (blood cultures negative), for which empirical Imipenem was started. Despite all taken measures, our patient unfortunately died due to septic shock. Bacterial examination of the dialysis unit water system and equipment as well as of the ventilator used by the patient was negative and no other cases of Cupriavidus pauculus infection were reported in our unit.

\section{Discussion}

This report demonstrates a complex example regarding both graft and patient outcomes following a priority kidney re-transplant context with some particularities we consider crucial to be addressed and discussed. Regarding renal outcomes, despite normal US and renal scintigraphy suggesting only ATN, the graft never gained function and our patient remained dialysis dependent (first PD and then CVVHDF). This was interpreted as delayed graft function (DGF) probably in the context of ATN (no biopsy was performed) which was, then, complicated with hemodynamic instability in the context of the infectious events. The absence of graft function aligned with the urgent need for immunosuppressive drug suspension in the context of severe infection prompted graft nephrectomy. Hence, no DGF biopsy was timely taken and, consequently, none of the main possible differential diagnoses, such as acute rejection, could be confirmed. Regarding patient's infectious outcomes, the presence of enteric microorganisms in peritoneal effluent (Enteroccocus faecium and Serratia marcescens), refractory to Tenckoff removal and directed antibiotic therapy, suggested bowel perforation-and confirmed in a tomography scan. Taking in consideration our patient's history of EPS, this complication could be expected as it has previously been described in literature. ${ }^{14,15}$

We present a rare case of a respiratory tract infection associated with Cupriavidus pauculus in a post-kidney-transplant patient. As discussed before, C. pauculus can cause infections either in hospitalized immunocompromised and immunocompetent patients. ${ }^{4,5,9}$ As risk factors, our patient was extremely immunodepressed (end-stage renal disease who got through aggressive induction and maintenance immunosuppressive therapy) alongside with other comorbidities. In this particular case, medical devices such as hemodialysis circuit equipment and the patient's ventilator (sources previously reported in literature) could represent the origin of contamination. ${ }^{6,12}$ However, all equipment samples collected did not grow any organism in this case, suggesting that no environmental contamination was involved. Concerning the treatment approach, isolation of C. pauculus from a clinical specimen is generally treated with high index of suspicion and usually regarded as contamination unless proven otherwise. ${ }^{16}$ It has been implicated in pseudo-outbreaks, but may be considered a pathogen if isolated from a sterile body fluid and correlated with clinical condition. ${ }^{16}$ In our case, since Cupriavidus pauculus was isolated in more than one sputum sample in a patient with respiratory tract signs and symptoms, this finding suggested that the agent could be causing a true infection, not just a contamination, and empirical imipenem was started. Few data are known about the optimal regimen for therapy of C. pauculus. Wide susceptibility to fluoroquinolones, ceftazidime and imipenem had been observed, for instance, Vay reported a meropenem-and imipenem-susceptible $C$. pauculus bacteremia. ${ }^{4,6}$ However, resistance to carbapenems has also been described. Salar et al., ${ }^{10}$ reported a case of imipenem-resistant $C$. pauculus bacteremia and Uzodi et al., ${ }^{17}$ related a meropenem-resistant C. pauculus bacteremia in a child on extracorporeal membrane oxygenation. There is also some data about colistin-sensitivity, such as the one described by Aydin et al.

In conclusion, we present a severe and rare case of a respiratory tract infection associated with Cupriavidus pauculus which, to our knowledge, is the first one to be described in a post-kidney-transplant patient. As ubiquitous as it is, the isolation of this uncommon agent should be carefully considered and treated as a pathogen if clinically suspected and environmental samples should always be collected from potential suspected nosocomial sources.

\section{Compliance with ethical standards}

This article does not contain any studies with human participants or animals performed by any of the authors.

\section{Informed consent}

Informed consent was obtained for all individual participants included in the study.

\section{Acknowledgments}

None.

\section{Conflicts of interest}

The authors have declared that no conflict of interest exists. 


\section{References}

1. Vandamme PJ, Goris T, Coenye B, et al. Assignment of Centers for Disease Control group IVc-2 to the genus Ralstonia as Ralstonia paucula sp. nov. Int J Syst Bacteriol. 1999;49(Pt 2):663-669.

2. Kalka-Moll WM, LiPuma JJ, Accurso FJ, et al. Airway infection with a novel Cupriavidus species in persons with cystic fibrosis. J Clin Microbiol. 2009;47(9):3026-3028.

3. Almasy E, Szederjesi J, Rad P, et al. A Fatal Case of Community Acquired Cupriavidus Pauculus Pneumonia. J Crit Care Med (Targu Mures). 2016;2(4):201-204.

4. D'Inzeo T, Santangelo R, Fiori B, et al. Catheter-related bacteremia by Cupriavidus metallidurans. Diagn Microbiol Infect Dis. 2015;81(1):9-12

5. Yahya R, Alyousef W, Omara A, et al. First case of pneumonia caused by Cupriavidus pauculus in an infant in the Gulf Cooperation Council. $J$ Infect Dev Ctries. 2017;11(2):196-198.

6. Vay C GS, Alperovich G, Almuzara M, et al. Bacteremia due to Cupriavidus pauculus (for- merly CDC Group IVc-2) in a hemodialysis patient. Clinical Microbiology Newsletter. 2007;29(4):30-32.

7. Aydin B, Dilli D, Zenciroglu A, et al. A case of newborn with community acquired pneumonia caused by Cupriavidus pauculus. Tuberk Toraks. 2012;60(2):160-162.

8. Yabuuchi E, Kosako Y, Yano I, et al. Transfer of two Burkholderia and an Alcaligenes species to Ralstonia gen. Nov.: Proposal of Ralstonia pickettii (Ralston, Palleroni and Doudoroff 1973) comb. Nov., Ralstonia solanacearum (Smith 1896) comb. Nov. and Ralstonia eutropha (Davis 1969) comb. Nov. Microbiol Immunol. 1995;39(11):897-904.
9. Anderson RR, Warnick P, Schreckenberger PC. Recurrent CDC group IVc-2 bacteremia in a human with AIDS. $J$ Clin Microbiol. 1997;35(3):780-782.

10. Uzodi AS, Schears GJ, Neal JR, et al. Cupriavidus pauculus bacteremia in a child on extracorporeal membrane oxygenation. ASAIO J. 2014;60(6):740-741.

11. Stovall SH, Wisdom C, McKamie W, et al. Nosocomial transmission of Cupriavidus pauculus during extracorporeal membrane oxygenation. ASAIO J. 2010;56(5):486-487.

12. Assbakan MS YT, Aydemir S, Bacakoğlu F. A case of ventilatorassociated pneumonia caused by Cupriavidus pauculus. Mikrobiyol Bul. 2010;44(1):127-131

13. Bianco G, Audisio E, Cavallo R, et al. Septic shock due to meropenemand colistin-resistant Cupriavidus pauculus. Journal of Hospital Infection. 2018;99(3):364-365.

14. Danford CJ LS, Smith MP, Wolf JL. Encapsulating peritoneal sclerosis World J Gastroenterol. 2018;24(28):3101-3111.

15. Morishita K, Otomo Y, Aiboshi J, et al. Encapsulating Peritoneal Sclerosis Complicated by an Intra-abdominal Abscess. Am J Kidney Dis. 2011;58(2):325-328

16. Duggal S, Gur R, Nayar R, et al. Cupriavidus pauculus (Ralstonia paucula) concomitant meningitis and septicemia in a neonate: first case report from India. Indian J Med Microbiol. 2013;31(4):405-409.

17. Salar A, Carratala J, Zurita A, et al. Bacteremia caused by CDC group IV c-2 in a patient with acute leukemia. Haematologica. 1998;83(7):670-672. 